\title{
Identification of a natural product-like STAT3 dimerization inhibitor by structure-based virtual screening
}

\author{
L-J Liu ${ }^{1,3}$, K-H Leung ${ }^{2,3}$, DS-H Chan ${ }^{2}$, Y-T Wang ${ }^{1}$, D-L Ma ${ }^{*, 2}$ and C-H Leung ${ }^{\star, 1}$
}

STAT3 regulates a variety of genes involved with cell proliferation, differentiation, apoptosis, angiogenesis, metastasis, inflammation, and immunity. The purpose of this study was to apply molecular docking techniques to identify STAT3 inhibitors from a database of over 90000 natural product and natural product-like compounds. The virtual screening campaign furnished 14 hit compounds, from which compound 1 emerged as a top candidate. Compound 1 inhibited STAT3 DNA-binding activity in vitro and attenuated STAT3-directed transcription in cellulo with selectivity over STAT1 and with comparable potency to the well-known STAT3 inhibitor S3I-201. Furthermore, compound 1 inhibited STAT3 dimerization and decreased STAT3 phosphorylation in cells without affecting STAT1 dimerization and phosphorylation. Compound 1 also exhibited selective anti-proliferative activity against cancer cells over normal cells in vitro. Molecular docking analysis suggested that compound 1 might putatively function as an inhibitor of STAT3 dimerization by binding to the SH2 domain. This study also validates the use of in silico techniques to identify inhibitors of protein-protein interactions, which are typically considered difficult to target with small molecules.

Cell Death and Disease (2014) 5, e1293; doi:10.1038/cddis.2014.250; published online 13 June 2014

Signal transducer and activator of transcription (STAT) proteins are a family of transcription factors that mediate gene expression in response to cytokines and growth factors. ${ }^{1}$ STAT3 regulates a variety of genes involved in cell proliferation, differentiation, apoptosis, angiogenesis, metastasis, inflammation, and immunity. ${ }^{2-4}$ Dysregulated STAT3 activity has been implicated in the development of a variety of solid and hematological tumors, including leukemia, lymphomas, and head and neck cancer. ${ }^{5-7}$ Additionally, elevated STAT3 levels have been associated with poor prognosis of certain cancers. ${ }^{8}$ The central role of aberrant STAT3 signaling in tumorigenesis has rendered STAT3, and, to a lesser extent, STAT5, as an attractive target in anticancer therapy, ${ }^{9,10}$ in addition to autoimmune and inflammatory applications. ${ }^{11-14}$

The structure of STAT3 is a characteristic of the STAT family, and includes an N-terminal coiled-coiled domain, a C-terminal transactivation domain, a DNA-binding domain, a Src homology $2(\mathrm{SH} 2)$ domain. The $\mathrm{N}$-terminal coiled-coiled domain is involved in protein-protein interactions for the formation of multiple types of dimer complexes, ${ }^{15}$ and also contains a lysine (Lys140) residue for methylation by histone methyl transferase SET9, which is a negative regulatory event. ${ }^{16}$ On the other hand, the $C$ terminus contains a conserved tyrosine (Tyr705) residue that is essential for
STAT3 activation. ${ }^{15}$ In the classical STAT3 activation pathway, growth factors and cytokines induce the tyrosine kinase activities of JAK or Src receptors, resulting in specific tyrosine phosphorylation of receptor chains. ${ }^{17}$ Latent STAT3 is recruited to the activated receptor via phosphotyrosine-SH2 recognition, and is phosphorylated at Tyr705 by JAK/Src. STAT3 monomers dimerize through reciprocal phosphotyrosine-SH2 interactions, and activated STAT3 dimers translocate into the nucleus where they bind to DNA response elements in the promoters of targeted genes, leading to gene transcription.

In normal cells, the activation of STAT3 is tightly regulated by a number of signaling mechanisms. ${ }^{8}$ Some protein tyrosine phosphatases (PTPs), such as the ubiquitously expressed SHP-2, contain SH2 domains that can potentially recognize the phosphotyrosine elements of any of the members of the STAT3 signaling pathway. ${ }^{18}$ In addition, the constitutively expressed protein inhibitor of activated STAT3 binds specifically to STAT3 and blocks its ability to bind to DNA and activate gene transcription. ${ }^{19}$ Finally, suppressors of cytokine signaling (SOCS) possess $\mathrm{SH} 2$ domains that recognize phosphotyrosine elements of JAKs, cytokine receptor chains and gp130. ${ }^{18}$ The versatile SOCS3, whose transcription is activated by STAT3 in a negative feedback mechanism, can

\footnotetext{
${ }^{1}$ State Key Laboratory of Quality Research in Chinese Medicine, Institute of Chinese Medical Sciences, University of Macau, Macao, China and ${ }^{2}$ Department of Chemistry, Hong Kong Baptist University, Kowloon Tong, Hong Kong, China

*Corresponding author: D-L Ma, Department of Chemistry, Room T1303, Cha Chi-ming Science Tower, Ho Sin Hang Campus, Hong Kong Baptist University, Kowloon Tong, Hong Kong, China. E-mail: edmondma@hkbu.edu.hk

or C-H Leung, State Key Laboratory of Quality Research in Chinese Medicine, Institute of Chinese Medical Sciences, University of Macau, Macao 999078 , China. Tel: + 8538397 8518; Fax: + 8532884 1358; E-mail: duncanleung@umac.mo

${ }^{3}$ These authors contributed equally to this work.

Abbreviations: STAT, signal transducer and activator of transcription; SH2, Src homology 2; PTPs, protein tyrosine phosphatises; PIAS3, protein inhibitor of activated STAT3; SOCS, suppressors of cytokine signaling; EGFR, human epidermal growth factor receptor; EGF, human epidermal growth factor; IFN- $\alpha$, interferon alpha protein; IFN- $\gamma$, interferon gamma protein; IL-6, interleukin 6; DMSO, dimethyl sulfoxide; VSL, virtual library screening; BPMC, biased probability Monte Carlo

Received 12.1.14; revised 03.5.14; accepted 06.5.14; Edited by A Stephanou
} 
block STAT3 signaling by the direct inhibition of JAK activity, by competing with STAT3 for phosphotyrosine residues on the receptor chains, or by binding to signaling proteins and triggering their proteasomal degradation. ${ }^{20}$

In contrast, constitutive STAT3 activation is a hallmark of a variety of human cancers. Though a large amount of evidence has been accumulated linking the pharmacological or genetic control of constitutively active STAT3 with tumor phenotype and progression in vivo, ${ }^{21,22}$ the precise mechanism of STAT3-induced tumor formation has yet to be completely established. Increased expression of human epidermal growth factor receptor (EGFR), Src or Src family of tyrosine kinases, or mutations in JAK proteins leading to hyperactive activity are factors that have been proposed to account for constitutively phosphorylated STAT3. ${ }^{23,24}$ The levels of STAT3-activating ligands such as TGF $\alpha$ and IL- 6 were also increased in the serum or tumors of patients with various types of human cancers. ${ }^{6}$ Alternatively, the disruption of epigenetic control of STAT3 regulators or the reduced expression of STAT3 antagonists, such as PTP, PIAS, or SOCS proteins may also promote excessive STAT3 activity, enhancing tumor growth. $^{3}$

Molecules that can inhibit STAT3 activity represent a potential avenue for chemotherapeutic intervention. ${ }^{25}$ Popular approaches include oligopeptides/peptidomimetics ${ }^{26}$ or G-quadruplex nucleic acids ${ }^{27,28}$ that target $\mathrm{SH} 2$ domains and disrupt STAT3 dimerization, or 'decoy' oligonucleotides that sequester active STAT3 and block the authentic STAT3-DNA interaction. ${ }^{29}$ More recently, there has been an increased interest in the development of small-molecule inhibitors of STAT3 activity. The amidosalicylic acid S3I-201 inhibits STAT3 dimerization and blocks STAT3 DNA-binding activity and STAT3-dependent transcription in vitro. ${ }^{22}$ Alternatively, platinum(IV) compounds have shown potent activity against STAT3 DNA-binding activity, STAT3-driven gene expression, and cancer growth, via binding to the STAT3 DNA-binding domain. ${ }^{30}$ Recently our group discovered the first example of a substitutionally-inert, Group 9 organometallic compound as a direct inhibitor of STAT3 dimerization with potent anti-tumor activities in an in vivo mouse xenograft model of melanoma. ${ }^{31}$ However, despite these promising studies, no compound discovered as a STAT3 inhibitor a priori has yet been approved for clinical use.

One challenge that has to be overcome by STAT3 inhibitors is the high degree of homology between the structures of STAT3 and STAT1, particularly in their SH2 domains. Bluyssen and co-workers ${ }^{32}$ have recently used comparative in silico docking to study the binding specificity of STAT inhibitors stattic and fludarabine, and have concluded that ligands targeting only the highly conserved phosphotyrosine binding pocket of the $\mathrm{SH} 2$ domain (i.e. stattic) will lack selectivity towards STATs, as STAT1 and STAT3 have identical active residues at this site. A number of compounds thought to be primarily STAT3 inhibitors, such as resveratrol, ${ }^{33}$ have also been found to inhibit STAT1 activity. Although STAT3 and STAT1 are highly similar in terms of both protein and target DNA sequences, they have different physiological effects. Deficiency in STAT1 leads to attenuation of interferon (IFN) responsiveness, increasing the susceptibility of mice to viral or bacterial infections. Moreover, activated STAT1 may behave as a tumor suppressor, ${ }^{34}$ though further studies are needed to elucidate this relationship. This suggests that selectivity for STAT3 over STAT1 may be a desirable characteristic for the development of novel anticancer therapeutics.

Structure-based virtual screening has emerged as an efficient strategy in drug discovery complementing conventional high-throughput techniques. ${ }^{35-39}$ By weeding out inactive non-binders in silico, the numbers of compounds to be synthesized and tested in vitro can be dramatically reduced. Meanwhile, natural products have historically represented an importance source of chemical scaffolds and bioactive substructures for the medicinal chemist. ${ }^{40} \mathrm{~A}$ number of natural products including curcumin, cucurbitacin, honokiol, guggulsterone, resveratrol, berbamine, and flavopiridol have been reported to exhibit STAT3 inhibitory activity. ${ }^{14}$ Encouraged by these ideas, we sought to apply molecular docking techniques to identify STAT3 inhibitors from a database of natural product and natural product-like compounds, and to evaluate the STAT3 inhibitory activity of the hit compounds in vitro.

\section{Results}

Virtual screening campaign and hit validation. The X-ray structure of the STAT3 homodimer bound to DNA (PDB: $1 B G 1)^{41}$ was used as the molecular model for our investigations. For the molecular docking, DNA was removed and only one of the monomers was used. The search area for screening was restricted to the $\mathrm{SH} 2$ domain of the STAT3 monomer. Over 90000 compounds from a chemical library of natural products and natural product-like molecules were screened in silico. Fourteen compounds (Figure 1) were screened in a preliminary ELISA to evaluate their ability to inhibit STAT3 DNA-binding activity in vitro (Figure 2). From these results, the benzofuran derivative compound 1 emerged as a top candidate.

Inhibition of STAT3 DNA-binding. A dose-response experiment was performed to determine the potency of compound 1 at inhibiting the DNA-binding activity of STAT3. In the assay, HepG2 extracts containing EGF-activated STAT3 were incubated with increasing concentrations of compound 1 or S3I-201. Encouragingly, a dose-dependent reduction in the DNA-binding activity of STAT3 was observed in the presence of compound 1. The potency of compound $1\left(\mathrm{IC}_{50}=\right.$ ca. $\left.15 \mu \mathrm{M}\right)$ was comparable to that of the positive control compound S3I$201\left(\mathrm{IC}_{50}=\right.$ ca. $\left.10 \mu \mathrm{M}\right)$ determined under the same conditions (Figure 3). We also performed a parallel experiment with the homologous STAT1 protein to evaluate the selectivity of compound 1. No inhibition in STAT1 DNA-binding was observed when COS-7 nuclear extracts containing IFN- $\gamma$ induced activated STAT1 were incubated in the presence of $30 \mu \mathrm{M}$ of compound and S3I-201 (Supplementary Figure S2). The selectivity of compound 1 (and S3I-201) for STAT3 over STAT1 was confirmed in HeLa nuclear extracts stimulated with IFN- $\alpha$, which phosphorylates STAT3 and STAT1 with comparable efficiency (Supplementary Figure S3). These results suggest that compound $\mathbf{1}$ is able to block the binding of activated STAT3 to its consensus DNA sequence in isolated 
<smiles>Cc1oc2ccc(OCC(=O)O)cc2c1C(=O)OC(C)CCl</smiles>

C1<smiles>COc1ccc(C(=O)Nc2ccccc2C(=O)NCCC(=O)O)cc1</smiles>

C2<smiles>Cn1c(=O)c2nc3ccc(NC(=O)/C=C/C(=O)O)cc3nc2n(C)c1=O</smiles><smiles>O=C(Cc1ccccc1)NC(CCCCCP(=O)(O)O)C(=O)O</smiles>

C3<smiles>COc1ccc(OCC(=O)Nc2ccc(C(=O)O)cc2NC(=O)COc2ccc(OC)cc2)cc1</smiles>

C4<smiles>O=C(O)c1ccc(NC(=O)C2Cc3ccccc3C(=O)O2)cc1O</smiles>

c9<smiles>O=C(O)CCNc1cc(C(=O)O)ccc1C(=O)O</smiles>

C5<smiles>Cc1ccc(S(=O)(=O)N[C@@H](C)C(=O)N2CCC(C(=O)N[C@@H](CC(=O)O)C(=O)O)CC2)cc1</smiles>

C6

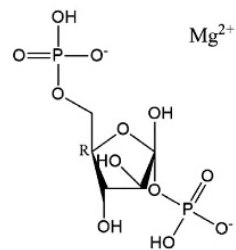

C11<smiles>COc1ccc2c(c1OC)C(=O)OC2Nc1ccc(C(=O)O)cc1</smiles><smiles>O=C(O)COc1ccc(C(=O)Cc2ccc3c(c2)OCCCO3)c(O)c1</smiles><smiles>COc1ccc(C=C2Oc3cc(OCC(=O)N[C@H](C(=O)O)C(C)O)ccc3C2=O)c(OC)c1OC</smiles>

C10

C12<smiles>O=C(O)COc1ccc2c(c1)c1ccnc3c4ccccc4c(=O)n2c13</smiles>

C13<smiles>COc1cccc(-c2nc(C(=O)N[C@@H](C)C(=O)O)cc3c2[nH]c2ccccc23)c1</smiles>

Figure 1 Structures of compounds 1-14 identified in the high-throughput virtual screening chosen for biological validation

nuclear extracts, and is selective for STAT3 over the closely related protein STAT1.

Molecular docking analysis. We performed molecular modeling of compound 1 with the STAT3 SH2 domain in order to further understand the mode of binding (Figure 4). Hydrogen bonds were predicted between the carboxylate functionality of compound 1 with Ser611 (1.99 A) and Glu612 (2.32 A), respectively, whereas the ether moiety of compound 1 formed a hydrogen bond to $\operatorname{Arg609}$ (2.06 A). According to the model, the benzofuran and isopropyl ester moieties do not significantly interact with the protein, with the closest distance between the benzofuran and Lys591 being 3.11 $\mathrm{A}$ and the closest distance between the isopropyl ester 


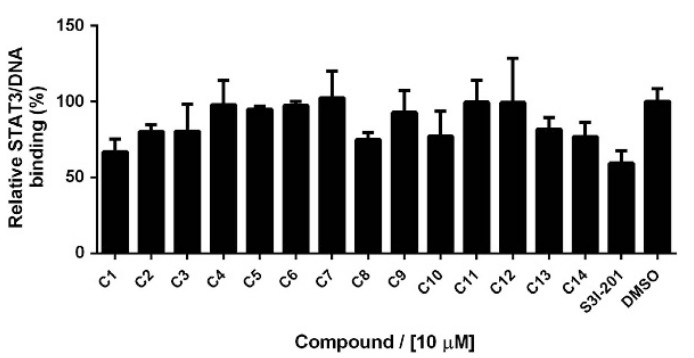

Figure 2 STAT3 DNA-binding inhibitory activity of compounds 1-14 as determined by ELISA. Microtitre plates coated with the STAT3 consensus sequence were incubated with nuclear extracts containing activated STAT3 and $10 \mu \mathrm{M}$ of compounds. STAT3 binding was detected using anti-STAT3 primary antibody and horseradish peroxidase-conjugated secondary antibody. Results are representative of three independent experiments

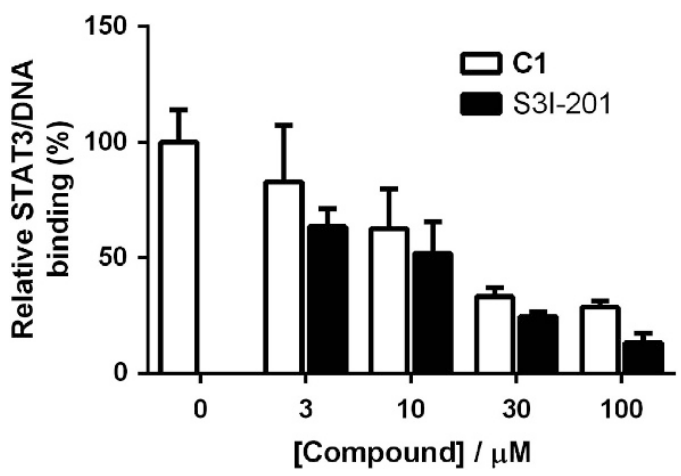

Figure 3 Compound 1 inhibits the DNA-binding activity of STAT3 in a dosedependent manner as measured by ELISA. Microtitre plates coated with the STAT3 consensus sequence were incubated with HepG2 nuclear extracts containing EGFactivated STAT3 and 1 or S3I-201 at the indicated concentrations. STAT3 binding was detected using anti-STAT3 primary antibody and horseradish peroxidaseconjugated secondary antibody. Results are representative of three independent experiments. Error bars represent the S.D. of triplicate results. Estimated $I_{50}$ values: compound 1: $15 \mu \mathrm{M}$, S3I-201: $10 \mu \mathrm{M}$

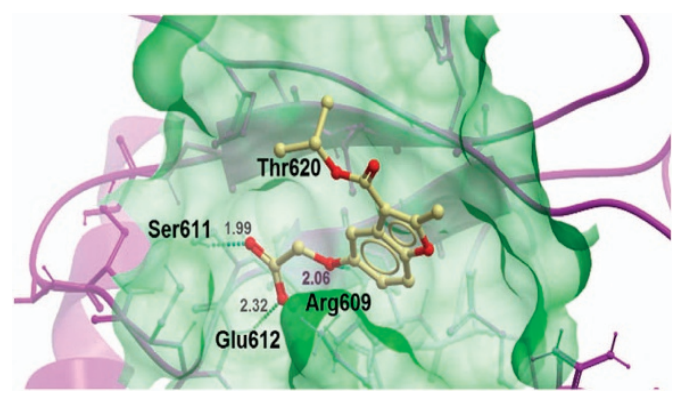

Figure 4 Low-energy-binding conformations of compound 1 bound to the STAT3 $\beta$ homodimer generated by virtual ligand docking. Compound 1 is depicted as a ball-and-stick model showing carbon (yellow) and oxygen (red) atoms. Hydrogen bonds are depicted as dotted lines. The binding pocket of the STAT3 $\beta$ is represented as a translucent green surface

and Thr620 being $5.83 \mathrm{~A}$. The binding score of -31.3 for compound 1 reflects the strong binding interaction between compound 1 and the STAT3 SH2 domain.

For further validation of the selectivity of compound $\mathbf{1}$, we performed molecular docking of compound $\mathbf{1}$ (Supplementary Figure S4) and S31-201 (Supplementary Figure S5) with STAT1 and STAT5 proteins. Surprisingly, the lowest-energy-binding poses of compound 1 in the $\mathrm{SH} 2$ domain of STAT1 and STAT5 were very different to that with STAT3 (Supplementary Figure S4). Compound 1 was predicted to form hydrogen-bonding interactions with Glu612 and Lys591 of STAT1 via its carboxylate group, but not with any of the residues of the STAT5 SH2 domain. The unfavorable binding scores of compound 1 bound to STAT1 $(-14.24)$ and STAT5 $(-15.1)$ are consistent with the results of the STAT3 DNA-binding experiment described above. Similarly, the binding modes of S3I-201 to STAT1, STAT3 and STAT5 were also different to each other (Supplementary Figure S5), which could possibly also account for the STAT3-specificity of this compound.

We also performed docking of a carboxylate derivative of compound 1 that could result from the cleavage of the isopropyl ester group by intracellular enzymes (Supplementary Figure S6). The lowest-energy-binding pose of the cleaved compound with STAT3 was almost identical to that exhibited by compound 1, though a less favorable binding score of -27.19 was obtained. Furthermore, several low-scoring molecules identified from the virtual screening campaign exhibited no STAT3 DNA-binding inhibitory activity, and were used as negative controls (Supplementary Figure S7). The docking scores of compounds $1-14$ against STAT1, STAT3 and STAT5 are summarized in Supplementary Table S1. Additionally, the lowest-energy-binding poses of compounds 1-14 within the STAT3 SH2 domain are presented in Supplementary Table S2.

Inhibition of STAT3-driven transcriptional activity in cells. Given that compound 1 was able to block STAT3 DNA-binding activity in a cell-free system, we next investigated the ability of compound $\mathbf{1}$ to antagonize STAT3-driven transcriptional activity in living cells. Transfected HeLa cells were incubated with compound 1 or S3I-201 and stimulated with EGF for $6 \mathrm{~h}$. Compound 1 attenuated STAT3-directed transcription in a dose-dependent fashion $\left(\mathrm{IC}_{50}=c a .30 \mu \mathrm{M}\right)$, as revealed by a reduction in the ratio of firefly to Renilla luciferase activity (Figure 5a). A comparative assay was performed using IFN- $\alpha$-stimulated HeLa cells transfected with plasmids bearing the STAT3 or STAT1 response element. Compound 1 selectively inhibited STAT3-directed transcriptional activity, while having negligible effect on STAT1-driven expression (Supplementary Figure S8). Notably, the potency of compound $\mathbf{1}$ was comparable to that of S31-201, which also showed selectivity for STAT3 over STAT1 under the same conditions. These results indicated that compound 1 could specifically inhibit STAT3-directed transcription in living cells, presumably due, at least in part, to the inhibition of STAT3 dimerization in the cytosol.

Inhibition of STAT3 dimerization. To further investigate the inhibition of STAT3 dimerization by compound $\mathbf{1}$ in cellulo, a co-immunoprecipitation assay was performed. HEK293T cells co-expressing STAT3-Flag and STAT3GFP were treated with the indicated concentrations of compound 1 and S3I-201 in a 6-well plate for $6 \mathrm{~h}$. Protein lysates were purified by anti-Flag magnetic beads, eluted with low $\mathrm{pH}$ buffer and analyzed by western blotting with antiFlag and anti-GFP antibodies. In the untreated cells, blotting 
a
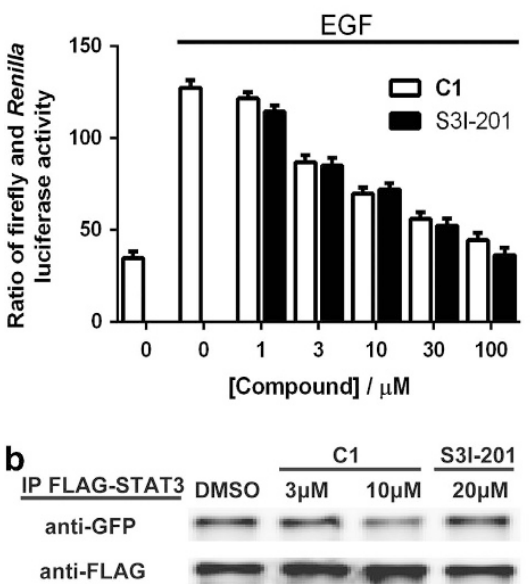

C

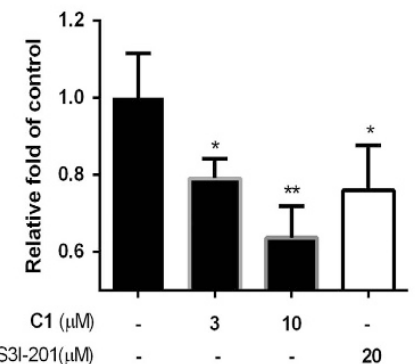

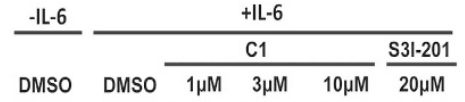

P-STAT3 (Y705)

STAT3

GADPH

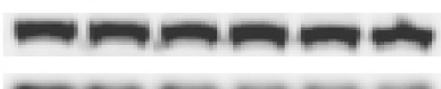

e

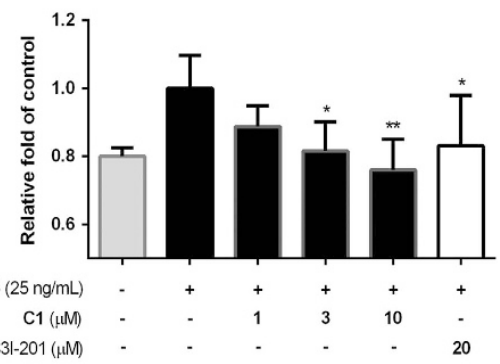

f

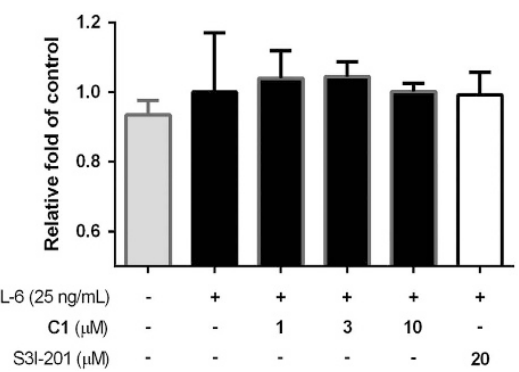

Figure 5 Effect of compound 1 on STAT3-driven transcription activity, STAT3 dimerization and STAT3 phosphorylation. (a) Compound 1 inhibits STAT3-driven transcription activity in EGF-stimulated HeLa cells as measured using a luciferase reporter assay. Estimated IC ${ }_{50}$ values: compound 1:30 $\mu \mathrm{M}, \mathrm{S} 3 \mathrm{I}-201: 30 \mu \mathrm{M}$. (b) Compound 1 inhibits the dimerization of STAT3 in HEK293T cells. (c) Quantification of STAT3 dimerization inhibition by densitometry analysis. (d) Compound 1 inhibits STAT3 Y705 phosphorylation but not total STAT3 content in HepG2 cells. (e) Quantification of STAT3 Y705 phosphorylation inhibition by densitometry analysis. (f) Densitometry analysis for the cell-based western blot shows no inhibition of compound 1 on total STAT3. Error bars represent the S.D. of triplicate results. ${ }^{\star} P<0.05$, ${ }^{\star \star} P<0.001$

of the immunoprecipitates with anti-GFP antibody revealed the presence of the STAT-GFP protein in the protein lysates, indicating that STAT3-GFP and STAT3-Flag proteins were pulled down together (Figures $5 b$ and $c$ ). However, the level of STAT3-GFP in the anti-Flag immunoprecipitates decreased as the concentration of compound 1 was increased. This result suggests that compound 1 disrupted STAT3-STAT3 dimerization in a dose-dependent manner in cellulo. To investigate the selectivity of compound 1 for STAT3 over STAT1 dimerization, HeLa cells were co-transfected with pRc/CMV-STAT3-Flag and pCMV6-AV-STAT3-GFP or pRc/CMV-STAT1-Flag and pcDNA-GFP-STAT1. The results showed that compound 1 selectively interfered with STAT3 dimerization but had no significant effect on STAT1 dimerization (Supplementary Figure S9).

Inhibition of phosphotyrosine-STAT3 levels. Phosphorylation of STAT3 at critical tyrosine residues is an essential requirement for STAT3 dimerization. Therefore, we investigated the effect of compound 1 on STAT3 tyrosine phosphorylation in cells. HepG2 cells were treated with the indicated concentrations of compound 1 for $6 \mathrm{~h}$ and stimulated with IL- 6 for $30 \mathrm{~min}$. Protein lysates were subjected to western blotting with anti-pY705-STAT3 antibody. The results revealed a dose-dependent decrease in IL-6-induced STAT3 phosphorylation as the concentration of compound 1 was increased
(Figures $5 d-f)$. However, compound 1 had no significant effect on total STAT3 expression. In a comparative assay, HeLa cells were treated with compound 1 and S3I-201 for $5 \mathrm{~h}$ and then stimulated with IFN- $\alpha$ for another $1 \mathrm{~h}$. Compound 1 reduced STAT3, but not STAT1, phosphorylation in a dosedependent manner (Figure 6). Moreover, the expression of STAT3 and STAT1 was not significantly reduced by treatment with compound $\mathbf{1}$. These results indicate that compound 1 could selectively decrease STAT3 phosphorylation over STAT1 phosphorylation in cellulo.

In vitro cytotoxicity. The cytotoxicity of compound $\mathbf{1}$ was investigated in LO2, HepG2, RAW264.7, and Caco2 cell lines. Cells were exposed to compound 1 (1-100 $\mu \mathrm{M})$ for $72 \mathrm{~h}$, and cellular proliferation was assessed using the MTT assay. Compound 1 was relatively nontoxic towards the normal liver LO2 cell line $\left(\mathrm{IC}_{50}>100 \mu \mathrm{M}\right)$, but showed dose-dependent inhibition of cellular proliferation of HepG2, RAW264.7 and Caco2 cell lines $\left(\mathrm{IC}_{50}=\mathrm{ca} .30 \mu \mathrm{M}\right)$ (Figure 7). Taken together, the data suggest that compound $\mathbf{1}$ displays marked selectivity for cancer cells over normal cells.

\section{Discussion}

Natural products offer a rich source of bioactive structural motifs for the development of new pharmaceuticals, and a 


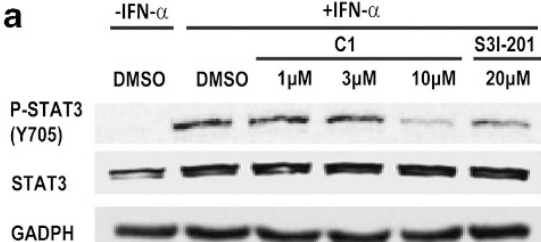

d

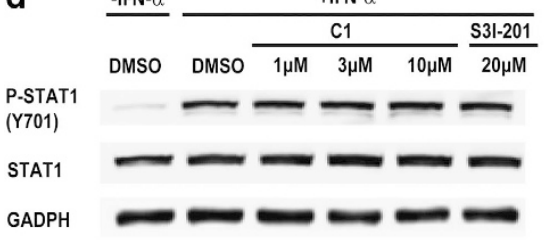

b

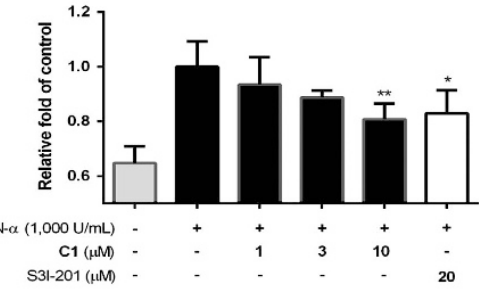

e

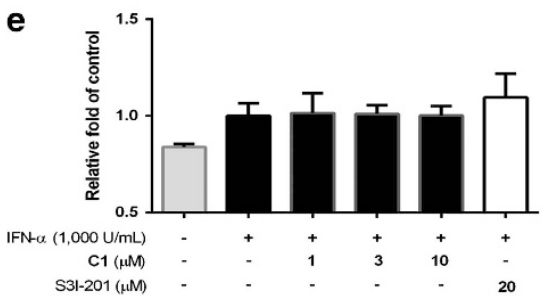

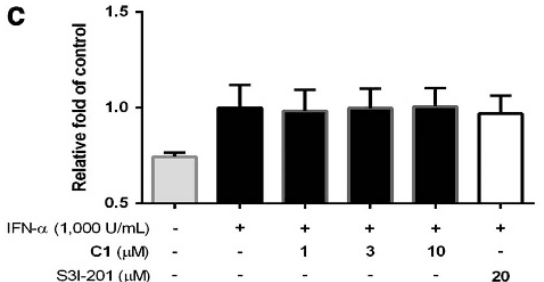

f

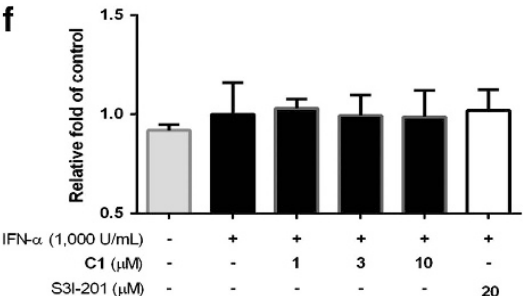

Figure 6 Effect of compound 1 on IFN- $\alpha$-induced STAT3 Y705 phosphorylation and IFN- $\alpha$-induced STAT1 Y701 phosphorylation in HeLa cells. (a) Compound 1 inhibits IFN- $\alpha$-induced STAT3 Y705 phosphorylation but not IFN- $\alpha$-induced total STAT3. (b) Quantification of STAT3 phosphorylation inhibition by densitometry analysis. (c) Quantification of total STAT3 expression by densitometry analysis. (d) Compound 1 has no effect on IFN- $\alpha$-induced STAT1 Y701 phosphorylation and IFN- $\alpha$-induced total STAT1. (e) Quantification of STAT1 phosphorylation inhibition by densitometry analysis. (f) Quantification of total STAT1 expression by densitometry analysis. Error bars represent the S.D. of triplicate results. ${ }^{*} P<0.05,{ }^{*} P<0.001$

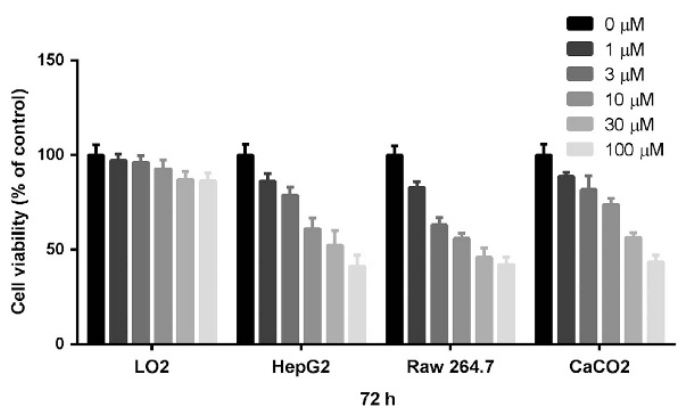

Figure 7 Cytotoxicity of compound 1 on cell viability as determined by the MTT assay. LO2, HepG2, RAW264.7, Caco2 were treated with the indicated concentrations of compound 1 for $72 \mathrm{~h}$. The data are expressed as the percentage of living cells compared with the negative control. Results are representative of three independent experiments. Error bars represent the S.D. of triplicate results

number of natural products have been reported in the literature to inhibit STAT3 activity. Curcumin, a natural product from the herb Curcuma long, inhibited JAK-STAT signaling and suppressed cell cycle arrest and cell invasion in vitro. ${ }^{42}$ Derivatives of the alkaloid berbamine were cytotoxic against melanoma cells harboring aberrant STAT3 activity, and decreased pSTAT3, pJAK2, and pSRC levels in those cells. ${ }^{43}$ Resveratrol, a widely studied polyphenolic compound found in variety of plants, inhibited constitutive and IL-6-induced STAT3 activity in multiple tumor cell types, leading to the decreased expression of anti-apoptotic proteins and the induction of apoptosis in vitro. ${ }^{44}$ Resveratrol and its derivatives have also been reported to suppress breast cancer cell proliferation via inhibition of STAT3 mediated MMP9 expression ${ }^{45}$ and to sensitize multiple myeloma, lung prostate, or pancreatic cancer cells to radiation or chemotherapeutic agents in vitro. ${ }^{46}$ However, despite many of these natural products reaching clinical status, the precise mechanism of STAT3 inhibition by these candidates has yet to be fully elucidated. ${ }^{14}$
In this study, the benzofuran derivative $\mathbf{1}$ was identified as a potential inhibitor of STAT3 dimerization using in silico screening. Benzofurans are common structural motifs present in numerous bioactive natural products and pharmaceuticals $^{47}$ owing to their diverse biological activities, such as antiangiogenic ${ }^{48}$ and antitumor ${ }^{49}$ properties. To the best of our knowledge, compound 1 has not been reported as a STAT3 inhibitor, and no biological activity of compound $\mathbf{1}$ has been presented in the literature.

In in vitro experiments, compound 1 inhibited STAT3 DNAbinding activity and reduced STAT3-driven luciferase activity in cells, but had no effect on IFN- $\alpha$ or IFN- $\gamma$-induced STAT1 DNA-binding activity or IFN- $\gamma$-induced STAT1-driven luciferase activity. Furthermore, compound $\mathbf{1}$ inhibited the interaction of STAT3-Flag and STAT3-GFP in protein lysates of treated cells, suggesting that the compound was able to antagonize STAT3 dimerization in cellulo, but had no effect on the interaction between STAT1-Flag and GFP-STAT1. Compound 1 also suppressed IL- 6 or IFN- $\alpha$-induced STAT3 Y705 phosphorylation in HepG2 cells and HeLa cells, respectively, but had no effect on IFN- $\alpha$-induced STAT1 phosphorylation in HeLa cells. Additionally, compound 1 showed no significant effect on total STAT3 or STAT1 expression. Finally, in the cell viability assay, compound 1 exhibited pronounced cytotoxicity against the neoplastic HepG2, RAW264.7, and Caco2 cell lines, but was benign towards the normal liver cell line LO2. A possible mechanism for the selective cytotoxicity for compound $\mathbf{1}$ towards cancer cells over normal cells could be due to the enhanced requirement for STAT3 activity in cancer cells, leading to a greater reduction in cell proliferation upon inhibition of STAT3 signaling by compound 1 .

Taken together, these data suggest that compound 1 acts as an inhibitor of STAT3 transcriptional activity through the disruption of STAT3 dimerization, which could contribute, at least in part, to the observed cytotoxicity of compound 1 . Based on the molecular modeling analysis, we anticipate that 
compound 1 may bind to the SH2 domain of STAT3 and block the STAT3 protein-protein interaction. Alternatively, the inhibition of STAT3 phosphorylation by compound 1 could also indirectly affect STAT3 dimerization. Further experiments will have to be performed to investigate the precise mechanism of STAT3 inhibitory activity of compound 1.

The binding of compound 1 to STAT3 was examined by molecular modeling. In the low-energy conformation of compound 1 in the $\mathrm{SH} 2$ domain, the small molecule was predicted to form hydrogen-bonding interactions with Ser611, Glu612, and Arg609. In the native protein, residues Arg609 and Ser611 are involved in direct polar interactions with phospho-Tyr705, and are, therefore, important elements of the reciprocal phosphotyrosine-SH2 interactions. ${ }^{41}$ Notably, the ester group of compound 1 is located near residue Thr620 in the ' $p Y+1$ ' binding sub-site, which is a region that has been noted to be more divergent among STATs. ${ }^{32}$ On the other hand, docking of compound 1 to STAT1 or STAT3 resulted in less favorable binding interactions and inferior binding scores. The distinct binding modes of compound 1 to STAT3 versus STAT1 or STAT5, despite their similar SH2 domains, provides a possible basis for the observed STAT3-specificity of compound 1. Other STAT3 small-molecule inhibitors in the literature that have been reported to inhibit STAT3 dimerization via binding to the SH2 domain include STA- $21^{50}$ and cryptotanshinone, ${ }^{51}$ which are in clinical trials for treatment of psoriasis $^{52}$ and polycystic ovary syndrome, ${ }^{53}$ respectively, as well as S3I-M2001, ${ }^{54}$ LLL12, ${ }^{55}$ FLLL32, ${ }^{56}$ S3I-201.1066, ${ }^{57}$ and BP-1-102. ${ }^{58}$ Very recently, the groups of Luk and Lunku have identified the natural product garcinol as an antagonist of STAT3 dimerization, via binding to the $\mathrm{SH} 2$ domain, and demonstrated its ability to inhibit human hepatocellular carcinoma growth in vitro and in vivo. ${ }^{59}$

As described in the introduction, the high degree of conservation in the $\mathrm{SH} 2$ domain of STAT proteins, including STAT3 and STAT1, makes the development of selective STAT inhibitors a difficult challenge. ${ }^{32}$ Owing to the different biological effects of STAT3 and STAT1, the requirement for STAT3-specificity has been thought to be important for the development of novel therapeutics agents. As a counterpoint, resveratrol $^{33}$ and curcumin, ${ }^{60}$ which have been shown to inhibit both STAT3 and STAT1, have progressed to clinical trials for a variety of malignancies, suggesting that absolute specificity for STAT3 is not an essential requirement for beneficial activity. In this study, compound $\mathbf{1}$ showed selectivity for STAT3 over STAT1 against STAT DNA-binding activity in vitro, as well as in terms of STAT-directed transcription, STAT dimerization, and STAT phosphorylation in cellulo. The selectivity of compound $\mathbf{1}$ for STAT3 was supported by the molecular modeling analysis, which predicted a lower binding score and a distinct binding interaction between compound 1 with the $\mathrm{SH} 2$ domain of STAT3 versus STAT1 or STAT5, including contact with the less-conserved $p Y+1$ region. This study also confirmed the selectivity of the inhibitor S3I-201 for STAT3 over STAT1, which was consistent with findings in previous studies. ${ }^{22,61}$

In conclusion, we have utilized structure-based virtual screening to discover a natural product-like inhibitor of STAT3. Compound 1 was able to inhibit STAT3 DNA-binding activity in a cell-free system and STAT3-driven luciferase expression in living cells, with potencies comparable to the well-known STAT3 inhibitor S3I-201. Additionally, compound 1 antagonized STAT3 dimerization and STAT3 tyrosine phosphorylation in cellulo. Our molecular modeling analysis suggested that compound 1 might putatively function as an inhibitor of STAT3 dimerization. This study also validates the use of structure-based molecular docking to discover novel inhibitors of protein-protein interactions, which are typically considered difficult to target with small molecules. We envisage that compound $\mathbf{1}$ may be employed as a useful scaffold for the development of more potent STAT3 dimerization inhibitors.

\section{Materials and Methods}

Cells and reagents. TransAM Transcription Factor ELISA was obtained from Active Motif (Carlsbad, CA, USA). Dual-Luciferase Reporter Assay System and pRL-TK vector were purchased from Promega (Madison, WI, USA). pLuc-TK/ STAT3 was purchased from Beyotime (Shanghai, Chian). Human epidermal growth factor (EGF) was purchased from BioVision Inc. (Mountain View, CA, USA). Interferon gamma protein (IFN- $\gamma$ ) was purchased from Abcam (Cambridge, MA, USA). Interleukin 6 (IL-6) was obtained from Sino Biological Inc. (Beijing, China). All antibodies were purchased from Cell Signaling Technology (Beverly, MA, USA). HeLa cells and HepG2 cells were cultured in DMEM containing $10 \%$ fetal bovine serum were incubated at $37^{\circ} \mathrm{C} / 5 \% \mathrm{CO}_{2}$ and maintained at a cell density of $0.2-1 \times 10^{6}$ cells $/ \mathrm{ml}$. HeLa cells were generously provided by Wendy Yeung (The Hong Kong University of Science and Technology, Hong Kong). COS7 cells and HEK293T cells were gifts from Ming-Yuen Lee (The University of Macau, Macao). All the compounds were dissolved in dimethyl sulfoxide (DMSO).

STAT3 and STAT1 transcription factor ELISA. The STAT3 and STAT1 DNA-binding assay was performed using TransAM Transcription Factor ELISA (Active Motif) according to the manufacturer's instructions. Briefly, HepG2 cells were stimulated with EGF $(100 \mathrm{ng} / \mathrm{ml})$ for activation of STAT3 for $15 \mathrm{~min}$. COS-7 cells were stimulated with IFN- $\gamma(25 \mathrm{ng} / \mathrm{ml})$ for activation of STAT1 for $1 \mathrm{~h} .{ }^{62} \mathrm{HeLa}$ cells were stimulated with IFN- $\alpha(1000 \mathrm{U} / \mathrm{ml})$ for activation of STAT3 and STAT1 for $1 \mathrm{~h}$. Cells were harvested and nuclear extract $(1 \mu \mathrm{g})$ containing activated STAT3/STAT1 was added with compound $(20 \mu \mathrm{l})$ and complete binding buffer $(30 \mu \mathrm{l})$ to microtitre wells coated with the STAT3 consensus sequence. The mixture was incubated at room temperature for $1 \mathrm{~h}$. The wells were washed three times with $1 \times$ wash buffer, and incubated with STAT3/STAT1 $\alpha$ antibody for $1 \mathrm{~h}$. The wells were washed as before and incubated with horseradish peroxideconjugated secondary antibody at room temperature for $1 \mathrm{~h}$. The wells were washed as before, incubated with $100 \mu \mathrm{l}$ of developing solution, quenched with $100 \mu \mathrm{l}$ stop solution, and the absorbance was measured at $\lambda=450 \mathrm{~nm}$.

Dual luciferase reporter assay. HeLa cells were co-transfected with pSTAT3-TA-luc (Beyotime) and pRL-TK (Promega) as a transfection efficiency control in a 6-well plate. After $12 \mathrm{~h}$, cells were transferred to a 48-well plate with complete medium and cultured for $24 \mathrm{~h}$. Cells were pre-treated with different concentration of compounds (in $0.05 \%$ DMSO) for $6 \mathrm{~h}$ in low FBS medium, and EGF $(100 \mathrm{ng} / \mathrm{ml})$ was subsequently added into the wells for $30 \mathrm{~min}$ to stimulate STAT3 expression and the cells were incubated for $30 \mathrm{~min}$. In the IFN- $\alpha$-induced comparative assay, HeLa cells were co-transfected with p-GAS-TA-luc (Biovector, Beijing, China) and pRL-TK in a 24-well plate for $6 \mathrm{~h}$. Cell were cultured with complete medium for another $18 \mathrm{~h}$. The indicated concentrations of compound 1 and S3I-201 were added to each well for $5 \mathrm{~h}$, followed by the addition of $1000 \mathrm{U} / \mathrm{ml}$ IFN- $\alpha$ for $1 \mathrm{~h}$ to stimulate STAT3/STAT1 expression. Cell lysates were collected according to the dual luciferase assay protocol (Promega). Sample light output was analyzed using a luminometer and the resulting data were normalized relative to $\mathrm{PRL}-\mathrm{TK}$ values.

Co-immunoprecipitation assay to evaluate STAT3 and STAT1 dimerization. HEK293T cells were co-transfected with pRc/CMV-STAT3-Flag (Addgene, Cambridge, MA, USA) and PCMV6-AC-STAT3-GFP (OriGene, Rockville, MD, USA). in a 6-well plate for $6 \mathrm{~h}$. In the IFN- $\alpha$-induced comparative assay, HeLa cells were co-transfected with pRc/CMV-STAT1-Flag (Addgene) and pcDNA-GFP-STAT1 (Addgene) in a 6-well plate for $6 \mathrm{~h}$. Cells were cultured in 
complete medium for another $12 \mathrm{~h}$, and then treated with compounds (in $0.05 \%$ DMSO) or vehicle control in low FBS medium for an additional $6 \mathrm{~h}$. Cell lysates were harvested and $30 \mu \mathrm{g}$ of each protein sample was pulled down with ANTIFLAG M2 magnetic beads (Sigma-Aldrich, St. Louis, MO, USA) according to the manufacturer's protocol. The purified protein samples were denatured for $5 \mathrm{~min}$ and subsequently subjected to SDS-PAGE. The protein samples were then transferred to a PVDF membrane and blocked with $5 \%$ non-fat dry milk in TBST for $1 \mathrm{~h}$ at room temperature. The membranes were then blotted with anti-Flag (Sigma-Aldrich) and anti-GFP (OriGene) antibody at $4{ }^{\circ} \mathrm{C}$ for overnight at a dilution of $1: 1000$. The membranes were washed three times and blotted with secondary antibodies at a dilution of $1: 10000$ at room temperature for $1 \mathrm{~h}$. After washing for $30 \mathrm{~min}$, membranes were analyzed with enhanced chemiluminescent Plus reagents (GE Healthcare, Waukesha, WI, USA).

Western blot analysis of STAT3 and STAT1 phosphorylation. HepG2 cells were seeded in a 6-well plate at a density of $5 \times 10^{5} \mathrm{ml}^{-1}$ in complete medium and cultured for $12 \mathrm{~h}$. Cells were then pre-treated with compounds (in $0.05 \%$ DMSO) or vehicle control in low-FBS medium for $6 \mathrm{~h}$. IL- $6(25 \mathrm{ng} / \mathrm{ml})$ was added to the wells to induce the phosphorylation of STAT3.$^{63,64}$ After $30 \mathrm{~min}$, cells were harvested and protein samples were quantified by the Pierce BCA Protein Assay Kit. $30 \mu \mathrm{g}$ of each protein sample was separated by SDS-PAGE. In the IFN- $\alpha$-induced comparative assay, HeLa cells were seeded in a 6-well plate at a density of $5 \times 10^{5} \mathrm{ml}^{-1}$ in complete medium and cultured for $12 \mathrm{~h}$. Cells were then pre-treated with compounds (in $0.05 \%$ DMSO) or vehicle control in low-FBS medium for $6 \mathrm{~h}$. IFN- $\alpha(1000 \mathrm{U} / \mathrm{ml})$ was added to the wells to induce the phosphorylation of STAT3 and STAT1. After $1 \mathrm{~h}$, cells were harvested and proteins samples were quantified. $30 \mu \mathrm{g}$ of each protein sample was separated by SDS-PAGE. The proteins were transferred to a PVDF membrane, and blocked with $5 \%$ non-fat dry milk in TBST for $1 \mathrm{~h}$ at room temperature. The membranes were incubated with primary antibodies (pY705-STAT3, pY701-STAT1, STAT3, STAT1 and GAPDH antibodies, Cell Signaling Technology) overnight. After treatment with secondary antibodies, membranes were analyzed with enhanced chemiluminescent Plus reagents (GE Healthcare).

Cell viability assay. LO2, HepG2, RAW264.7, Caco2 cells were seeded at 4000 cells per well in a 96-well culture microplate and incubated overnight at $37^{\circ} \mathrm{C}$. Serial dilutions of compound 1 were added to each well and the microplate was incubated at $37^{\circ} \mathrm{C}, 5 \% \mathrm{CO}_{2}, 95 \%$ air in a humidified incubator for $72 \mathrm{~h}$. Hundred microliter of MTT (3-(4,5-dimethylthiazol-2-yl)-2,5-tetrazolium bromide) reagent $(1 \mathrm{mg} / \mathrm{ml})$ was added to each well. After $4 \mathrm{~h}$, the medium was replaced with $100 \mu \mathrm{l} \mathrm{pf} \mathrm{DMSO}$ and the wells were incubated at room temperature for $10 \mathrm{~min}$ with shaking. Color intensity was measured at $570 \mathrm{~nm}$ using a microplate reader. The $\mathrm{IC}_{50}$ value of compound 1 was determined by the dose dependence of the surviving cells after exposure to 1 for $72 \mathrm{~h}$.

\section{Molecular docking and virtual screening}

Model construction: The initial model of STAT3 was derived from the X-ray crystal structure of the STAT3 homodimer bound to DNA (PDB: 1BG1), ${ }^{22}$ using the molecular conversion procedure implemented in the ICM-pro 3.6-1d program (Molsoft, San Diego, CA, USA). ${ }^{65}$ The molecular conversion procedure implemented in ICM-pro 3.6-1d program can read, build, convert, refine, analyze, and superimpose molecules and provide target evaluation to generate three dimensional models. DNA was removed and only one of the monomers was used. Hydrogen and missing heavy atoms were added to the receptor structure, and atom types and partial charges were assigned. The model was then subjected to local energy minimization to identify the optimal position by using the ICM biased probability Monte Carlo algorithm ${ }^{66}$ and analytical derivatives in the internal coordinates. The optimization gradient was $0.05 \mathrm{kcal} / \mathrm{M} / \mathrm{radian}^{66}$ For the selectivity analysis, X-ray crystal structures of STAT1 (PDB: 1BF5) and STAT5 (PDB: 1Y1U) were processed in a similar fashion.

High-throughput molecular docking: A chemical library containing over 90000 natural product or natural product-like compounds (ZINC natural product database) was docked to the molecular model of STAT3 in silico. Molecular docking was performed using the virtual library screening module in the ICM-Pro 3.6-1d program (Molsoft). In the ICM fast docking and VLS procedure, the receptor all-atom model was converted into energy potential maps calculated on a fine $3 \mathrm{D}$ grid $(0.5 \mathrm{~A}$ cell). The grid potential maps account for van der Waals, hydrogen bonding, hydrophobic, and electrostatic interactions between ligand and receptor. The search area for molecular docking was restricted to the SH2 domain of the STAT3 monomer. Each compound in the library was assigned the MMFF ${ }^{67}$ force field atom types and charges and was then subjected to Cartesian minimization. During the docking analysis, the ligand was represented by an all-atom model and considered fully flexible in the potential field of the receptor, and the binding pose and internal torsions were sampled by the biased probability Monte Carlo (BPMC) minimization procedure, which involved local energy minimization after each random move. Each compound was docked to the protein complex binding pocket, and a score from the docking was assigned to each compound according to the weighed component of the ICM scoring function (see below). Each compound was docked three times to ensure the convergence of the Monte Carlo optimization, and the minimum score of each ligand from the three independent docking experiments was retained and used for ranking. The docking procedure takes about $30 \mathrm{~s}$ of time per compound on a Intel Xeon $2.8 \mathrm{GHz}$ CPU using a 100 processor Linux cluster. A permissive cutoff score of -30.0 was chosen in order to weed out potential non-binding ligands and to reduce the size of compounds tested in vitro. The binding score distribution of all the compounds is depicted in Supplementary Figure S1. Fourteen compounds were purchased for in vitro biological testing.

ICM full-atom ligand-receptor complex refinement and scoring: Once the ligand-receptor complexes are generated by molecular docking, they have to be subjected to complex refinement and scoring. According to the ICM method, ${ }^{68}$ the molecular system was described using internal coordinates as variables. Energy calculations were based on the ECEPP/3 force field with a distancedependent dielectric constant. The BPMCminimization procedure was used for global energy optimization. This procedure consisted of four iterative steps. The BPMC global-energy-optimization method consists of (1) a random conformation change of the free variables according to a predefined continuous probability distribution; (2) local energy minimization of analytical differentiable terms; (3) calculation of the complete energy including non-differentiable terms such as entropy and solvation energy; (4) acceptance or rejection of the total energy based on the Metropolis criterion and return to step (1). The binding between the small molecules and STAT3 were evaluated with a full-atom ICM ligand binding score ${ }^{69}$ from a multi-receptor screening benchmark as a compromise between approximated Gibbs free energy of binding and numerical errors. The scoring function should give a good approximation of the binding free energy between a ligand and a receptor and is usually a function of different energy terms based on a force field. The ICM scoring function is weighted according to the following parameters (i) internal force field energy of the ligand, (ii) entropy loss of the ligand between bound and unbound states, (iii) ligand-receptor hydrogen bond interactions, (iv) polar and nonpolar solvation energy differences between bound and unbound states, (v) electrostatic energy, (vi) hydrophobic energy, and (vii) hydrogen bond donor or acceptor desolvation. The lower the ICM score, the higher the chance the ligand is a binder. The score was calculated by:

$S_{\text {bind }}=E_{\mathrm{int}}++T \Delta S_{\mathrm{Tor}}++E_{\mathrm{vw}}++\alpha_{1} E_{\mathrm{el}}++\alpha_{2} E_{\mathrm{hb}}++\alpha_{3} E_{\mathrm{hp}}++\alpha_{4} E_{\mathrm{sf}}$

where $E_{\mathrm{vw}}, E_{\mathrm{el}}, E_{\mathrm{hb}}, E_{\mathrm{hp}}$, and $E_{\mathrm{sf}}$ are van der Waals, electrostatic, hydrogen bonding, and nonpolar and polar atom solvation energy differences between bound and unbound states, respectively. $E_{\text {int }}$ is the ligand internal strain, $\Delta S_{\text {Tor }}$ is its conformational entropy loss upon binding, and $T=300 \mathrm{~K}$, and $\alpha_{\mathrm{i}}$ are ligandand receptor-independent constants. ${ }^{70}$

Data analysis. All data were reported as the means of at least three separate experiments. Group comparisons between the control group and various drug treatment groups were done by a one-way ANOVA using GraphPad Prism software (Prism). Significance was considered if a $P$-value of $<0.05$ was reached. Densitometry of western blots was performed by scanning of the exposed film and using Quantity One analysis software (Bio-Rad, Richmond, CA, USA).

\section{Conflict of Interest}

The authors declare no conflict of interest.

Acknowledgements. This work is supported by Hong Kong Baptist University (FRG2/12-13/021 and FRG2/13-14/008), Centre for Cancer and Inflammation Research, School of Chinese Medicine (CCIR-SCM, HKBU), the Health and Medical Research Fund (HMRF/13121482), the Research Grants Council (HKBU/ 201811, HKBU/204612 and HKBU/201913), the French National Research 
Agency/Research Grants Council Joint Research Scheme (A-HKBU201/12), the Science and Technology Development Fund, Macao SAR (103/2012/A3, 001/2012/ A) and the University of Macau (MYRG091(Y3-L2)-ICMS12-LCH, MYRG121(Y3L2)-ICMS12-LCH, MRG007/LCH/2014/ICMS and MRG023/LCH/2013/ICMS).

1. Sehgal PB. Paradigm shifts in the cell biology of STAT signaling. Semin Cell Dev Biol 2008; 19: $329-340$

2. Levy DE, Inghirami G. STAT3: a multifaceted oncogene. Proc Natl Acad Sci USA 2006; 103: 10151-10152.

3. Frank DA. STAT3 as a central mediator of neoplastic cellular transformation. Cancer Lett 2007: 251: 199-210.

4. Aggarwal BB, Kunnumakkara AB, Harikumar KB, Gupta SR, Tharakan ST, Koca C et al. Signal transducer and activator of transcription-3, inflammation, and cancer: how intimate is the relationship? Ann N Y Acad Sci 2009; 1171: 59-76.

5. Lai R, Rassidakis GZ, Medeiros LJ, Leventaki V, Keating M, McDonnell TJ. Expression of STAT3 and its phosphorylated forms in mantle cell lymphoma cell lines and tumours. J Pathol 2003; 199: 84-89.

6. Leeman RJ, Lui VW, Grandis JR. STAT3 as a therapeutic target in head and neck cancer Expert Opin Biol Ther 2006; 6: 231-241.

7. Furqan M, Mukhi N, Lee B, Liu D. Dysregulation of JAK-STAT pathway in hematological malignancies and JAK inhibitors for clinical application. Biomark Res 2013; 1: 5-14.

8. Johnston PA, Grandis JR. STAT3 signaling: anticancer strategies and challenges. Mol Interv 2011; 11: 18-26.

9. Darnell JE. Validating Stat3 in cancer therapy. Nat Med 2005; 11: 595-596.

10. Furqan M, Akinleye A, Mukhi N, Mittal V, Chen Y, Liu D. STAT inhibitors for cancer therapy. $J$ Hematol Oncol 2013; 6: 90-100.

11. Yu H, Pardoll D, Jove R. STATs in cancer inflammation and immunity: a leading role fo STAT3. Nat Rev Cancer 2009; 9: 798-809.

12. Fu XY. STAT3 in immune responses and inflammatory bowel diseases. Cell Res 2006; 16 214-219.

13. Egwuagu CE. STAT3 in CD4 + T helper cell differentiation and inflammatory diseases. Cytokine 2009; 47: 149-156.

14. Miklossy G, Hilliard TS, Turkson J. Therapeutic modulators of STAT signalling for human diseases. Nat Rev Drug Discov 2013; 12: 611-629.

15. Heinrich PC, Behrmann I, Haan S, Hermanns HM, Muller-Newen G, Schaper F. Principles of interleukin (IL)-6-type cytokine signalling and its regulation. Biochem J 2003; 374: 1-20.

16. Yang JB, Huang J, Dasgupta M, Sears N, Miyagi M, Wang BL et al. Reversible methylation of promoter-bound STAT3 by histone-modifying enzymes. Proc Natl Acad Sci USA 2010; 107: 21499-21504.

17. Murray PJ. The JAK-STAT signaling pathway: input and output integration. J Immuno 2007; 178: 2623-2629.

18. Wormald S, Hilton DJ. Inhibitors of cytokine signal transduction. J Biol Chem 2004; 279 821-824.

19. Shuai K. Regulation of cytokine signaling pathways by PIAS proteins. Cell Res 2006; 16 : 196-202.

20. Croker BA, Kiu H, Nicholson SE. SOCS regulation of the JAK/STAT signalling pathway. Semin Cell Dev Biol 2008; 19: 414-422.

21. Niu G, Heller R, Catlett-Falcone R, Coppola D, Jaroszeski M, Dalton W et al. Gene therapy with dominant-negative Stat3 suppresses growth of the murine melanoma B16 tumor in vivo. Cancer Res 1999; 59: 5059-5063.

22. Siddiquee K, Zhang S, Guida WC, Blaskovich MA, Greedy B, Lawrence HR et al. Selective chemical probe inhibitor of Stat3, identified through structure-based virtual screening, induces antitumor activity. Proc Natl Acad Sci USA 2007; 104: 7391-7396.

23. Silva CM. Role of STATs as downstream signal transducers in Src family kinase-mediated tumorigenesis. Oncogene 2004; 23: 8017-8023.

24. Alvarez JV, Greulich H, Sellers WR, Meyerson M, Frank DA. Signal transducer and activator of transcription 3 is required for the oncogenic effects of non-small-cell lung cancer-associated mutations of the epidermal growth factor receptor. Cancer Res 2006 66: 3162-3168.

25. Yue $P$, Turkson J. Targeting STAT3 in cancer: how successful are we? Expert Opin Invest Drugs 2009; 18: 45-56.

26. Turkson J, Ryan D, Kim JS, Zhang Y, Chen Z, Haura E et al. Phosphotyrosyl peptides block Stat3-mediated DNA binding activity, gene regulation, and cell transformation. J Biol Chem 2001; 276: 45443-45455.

27. Jing N, Zhu Q, Yuan P, Li Y, Mao L, Tweardy DJ. Targeting signal transducer and activator of transcription 3 with G-quartet oligonucleotides: a potential novel therapy for head and neck cancer. Mol Cancer Ther 2006; 5: 279-286.

28. Weerasinghe $P$, Garcia GE, Zhu Q, Yuan P, Feng L, Mao L et al. Inhibition of Stat3 activation and tumor growth suppression of non-small cell lung cancer by G-quartet oligonucleotides. Int J Oncol 2007; 31: 129-136.

29. Xi S, Gooding WE, Grandis JR. In vivo antitumor efficacy of STAT3 blockade using a transcription factor decoy approach: implications for cancer therapy. Oncogene 2005; 24 970-979.
30. Turkson J, Zhang S, Mora LB, Burns A, Sebti S, Jove R. A novel platinum compound inhibits constitutive Stat3 signaling and induces cell cycle arrest and apoptosis of malignant cells. J Biol Chem 2005; 280: 32979-32988.

31. Ma DL, Liu LJ, Leung KH, Chen YT, Zhong HJ, Chan DSH et al. Antagonising STAT3 Dimerization by a Rhodium(III) Complex. Angew Chem Int Ed Engl 2014; e-pub ahead of print 30 May 2014; doi:10.1002/anie.201404686.

32. Szelag M, Sikorski K, Czerwoniec A, Szatkowska K, Wesoly J, Bluyssen HA. In silico simulations of STAT1 and STAT3 inhibitors predict SH2 domain cross-binding specificity. Eur J Pharmacol 2013; 720: 38-48.

33. Li T, Wang W, Chen H, Li T, Ye L. Evaluation of anti-leukemia effect of resveratrol by modulating STAT3 signaling. Int Immunopharmacol 2010; 10: 18-25.

34. Regis G, Pensa S, Boselli D, Novelli F, Poli V. Ups and downs: The STAT1:STAT3 seesaw of Interferon and gp130 receptor signalling. Semin Cell Dev Biol 2008; 19 : 351-359

35. Lee HM, Chan DSH, Yang F, Lam HY, Yan SC, Che CM et al. Identification of natural product Fonsecin $\mathrm{B}$ as a stabilizing ligand of c-myc G-quadruplex DNA by high-throughput virtual screening. Chem Commun (Camb) 2010; 46: 4680-4682.

36. Chan DSH, Lee HM, Yang F, Che CM, Wong CCL, Abagyan R et al. Structure-based discovery of natural-product-like TNF-alpha inhibitors. Angew Chem Int Ed Engl 2010; 49: 2860-2864.

37. Leung $\mathrm{CH}$, Chan DSH, Yang H, Abagyan R, Lee SMY, Zhu GY et al. A natural product-like inhibitor of NEDD8-activating enzyme. Chem Commun (Camb) 2011; 47: 2511-2513.

38. Zhong HJ, Ma VPY, Cheng Z, Chan DSH, He HZ, Leung KH et al. Discovery of a natural product inhibitor targeting protein neddylation by structure-based virtual screening. Biochimie 2012; 94: 2457-2460.

39. Ma DL, Chan DSH, Leung $\mathrm{CH}$. Drug repositioning by structure-based virtual screening. Chem Soc Rev 2013; 42: 2130-2141.

40. Newman DJ, Cragg GM. Natural products as sources of new drugs over the 30 years from 1981 to 2010. J Nat Prod 2012; 75: 311-335.

41. Becker S, Groner B, Muller CW. Three-dimensional structure of the Stat3beta homodimer bound to DNA. Nature 1998; 394: 145-151.

42. Tu SP, Jin H, Shi JD, Zhu LM, Suo Y, Lu G et al. Curcumin induces the differentiation of myeloid-derived suppressor cells and inhibits their interaction with cancer cells and related tumor growth. Cancer Prev Res (Phila) 2012; 5: 205-215.

43. Nam S, Xie J, Perkins A, Ma Y, Yang F, Wu J et al. Novel synthetic derivatives of the natural product berbamine inhibit Jak2/Stat3 signaling and induce apoptosis of human melanoma cells. Mol Oncol 2012; 6: 484-493.

44. Shakibaei M, Harikumar KB, Aggarwal BB. Resveratrol addiction: to die or not to die. Mol Nutr Food Res 2009; 53: 115-128.

45. Kim JE, Kim HS, Shin YJ, Lee CS, Won C, Lee SA et al. LYR71, a derivative of trimeric resveratrol, inhibits tumorigenesis by blocking STAT3-mediated matrix metalloproteinase 9 expression. Exp Mol Med 2008; 40: 514-522.

46. Gupta SC, Kannappan R, Reuter S, Kim JH, Aggarwal BB. Chemosensitization of tumors by resveratrol. Ann N Y Acad Sci 2011; 1215: 150-160.

47. Yeung K-S. Furans and Benzofurans. Top Heterocycl Chem 2012; 29: 47-76.

48. Chen Y, Chen S, Lu X, Cheng H, Ou Y, Cheng H et al. Synthesis, discovery and preliminary SAR study of benzofuran derivatives as angiogenesis inhibitors. Bioorg Med Chem Lett 2009; 19: 1851-1854

49. Hayakawa I, Shioya R, Agatsuma T, Sugano Y. Synthesis and evaluation of 3-methyl4-oxo-6-phenyl-4,5,6,7-tetrahydrobenzofuran-2-carboxylic acid ethyl ester derivatives as potent antitumor agents. Chem Pharm Bull (Tokyo) 2005; 53: 638-640.

50. Song $\mathrm{H}$, Wang RX, Wang SM, Lin J. A low-molecular-weight compound discovered through virtual database screening inhibits Stat3 function in breast cancer cells. Proc Natl Acad Sci USA 2005; 102: 4700-4705.

51. Shin DS, Kim HN, Shin KD, Yoon YJ, Kim SJ, Han DC et al. Cryptotanshinone inhibits constitutive signal transducer and activator of transcription 3 function through blocking the dimerization in DU145 prostate cancer cells. Cancer Res 2009; 69: 193-202.

52. Miyoshi K, Takaishi M, Nakajima K, Ikeda M, Kanda T, Tarutani M et al. Stat3 as a therapeutic target for the treatment of psoriasis: a clinical feasibility study with STA-21, a Stat3 inhibitor. J Invest Dermatol 2011; 131: 108-117.

53. Shen WJ, Zhang YH, Li W, Cong J, Zhou Y, Ng EHY et al. Effects of tanshinone on hyperandrogenism and the quality of life in women with polycystic ovary syndrome: protocol of a double-blind, placebo-controlled, randomised trial. BMJ Open 2013; 3: 1-6.

54. Siddiquee KAZ, Gunning PT, Glenn M, Katt WP, Zhang S, Schroeck C et al. An oxazolebased small-molecule Stat3 inhibitor modulates Stat3 stability and processing and induces antitumor cell effects. ACS Chem Biol 2007; 2: 787-798.

55. Onimoe GI, Liu AG, Lin L, Wei CC, Schwartz EB, Bhasin D et al. Small molecules, LLL12 and FLLL32, inhibit STAT3 and exhibit potent growth suppressive activity in osteosarcoma cells and tumor growth in mice. Invest New Drugs 2012; 30: 916-926.

56. Fossey SL, Bear MD, Lin JY, Li CL, Schwartz EB, Li PK et al. The novel curcumin analog FLLL32 decreases STAT3 DNA binding activity and expression, and induces apoptosis in osteosarcoma cell lines. BMC Cancer 2011; 11: 112-126.

57. Zhang XL, Yue PB, Fletcher S, Zhao W, Gunning PT, Turkson J. A novel small-molecule disrupts Stat3 SH2 domain-phosphotyrosine interactions and Stat3-dependent tumor processes. Biochem Pharmacol 2010; 79: 1398-1409. 
58. Zhang XL, Yue PB, Page BDG, Li TS, Zhao W, Namanja AT et al. Orally bioavailable smallmolecule inhibitor of transcription factor Stat3 regresses human breast and lung cancer xenografts. Proc Natl Acad Sci USA 2012; 109: 9623-9628.

59. Sethi G, Chatterjee S, Rajendran P, Li F, Shanmugam MK, Wong KF et al. Inhibition of STAT3 dimerization and acetylation by garcinol suppresses the growth of human hepatocellular carcinoma in vitro and in vivo. Mol Cancer 2014; 13: 66.

60. Glienke W, Maute L, Wicht J, Bergmann L. Curcumin inhibits constitutive STAT3 phosphorylation in human pancreatic cancer cell lines and downregulation of survivin/ BIRC5 gene expression. Cancer Invest 2010; 28: 166-171.

61. Pang M, Ma L, Gong R, Tolbert E, Mao H, Ponnusamy M et al. A novel STAT3 inhibitor, S3l-201, attenuates renal interstitial fibroblast activation and interstitial fibrosis in obstructive nephropathy. Kidney Int 2010; 78: 257-268.

62. Darnell JE, Kerr IM, Stark GR. Jak-STAT pathways and transcriptional activation in response to IFNs and other extracellular signaling proteins. Science 1994; 264: 1415-1421.

63. Zhong Z, Wen Z, Darnell JE. Stat3: a STAT family member activated by tyrosine phosphorylation in response to epidermal growth factor and interleukin-6. Science 1994; 264: 95-98

64. Zhong Z, Wen ZL, Darnell JE. Stat3 and Stat4 - Members of the Family of Signal Transducers and Activators of Transcription. Proc Natl Acad Sci USA 1994; 91: 4806-4810.

65. Abagyan R, Orry A, Rausch E, Budagyan L, Totrov M. ICM Manual, 3.0, MolSoft LLCLa Jolla, CA, USA 2009

66. Abagyan R, Totrov M. Biased probability Monte Carlo conformational searches and electrostatic calculations for peptides and proteins. J Mol Biol 1994; 235: 983-1002.
67. Halgren TA. Merck molecular force field. I-V. J Comput Chem 1996; 17: 490-519.

68. Totrov M, Abagyan R. Flexible protein-ligand docking by global energy optimization in internal coordinates. Proteins 1997; Suppl 1: 215-220.

69. Schapira M, Totrov M, Abagyan R. Prediction of the binding energy for small molecules, peptides and proteins. J Mol Recognit 1999; 12: 177-190.

70. Totrov $M$, Abagyan $R$. Derivation of senstitive discrimination potential for virtual ligand screening. In:RECOMB '99: Third Annual International Conference on Computational Molecular Biology. Association for Computing Machinery: New York: Lyon, France 1999.

cc) $९$ Cell Death and Disease is an open-access journal published by Nature Publishing Group. This work is licensed under a Creative Commons Attribution-NonCommercialNoDerivs 3.0 Unported License. The images or other third party material in this article are included in the article's Creative Commons license, unless indicated otherwise in the credit line; if the material is not included under the Creative Commons license, users will need to obtain permission from the license holder to reproduce the material. To view a copy of this license, visit http://creativecommons.org/licenses/ by-nc-nd/3.0/

Supplementary Information accompanies this paper on Cell Death and Disease website (http://www.nature.com/cddis) 